\title{
Homogenization of metamaterials: Parameters retrieval methods and intrinsic problems
}

\section{Andryieuski, Andrei; Malureanu, Radu; Lavrinenko, Andrei}

\section{Published in:}

Proceedings of the International Conference on Transparent Optical Networks

Link to article, DOI:

10.1109/ICTON.2010.5549255

Publication date:

2010

Document Version

Publisher's PDF, also known as Version of record

Link back to DTU Orbit

Citation (APA):

Andryieuski, A., Malureanu, R., \& Lavrinenko, A. (2010). Homogenization of metamaterials: Parameters retrieval methods and intrinsic problems. In Proceedings of the International Conference on Transparent Optical Networks (pp. 1-5). IEEE. https://doi.org/10.1109/ICTON.2010.5549255

\section{General rights}

Copyright and moral rights for the publications made accessible in the public portal are retained by the authors and/or other copyright owners and it is a condition of accessing publications that users recognise and abide by the legal requirements associated with these rights.

- Users may download and print one copy of any publication from the public portal for the purpose of private study or research.

- You may not further distribute the material or use it for any profit-making activity or commercial gain

- You may freely distribute the URL identifying the publication in the public portal 


\title{
Homogenization of Metamaterials: Parameters Retrieval Methods and Intrinsic Problems
}

\author{
Andrei Andryieuski, Radu Malureanu, Andrei V. Lavrinenko* \\ Technical University of Denmark, Ørsteds Plads, Building 343, Kongens Lyngby, DK-2800, Denmark \\ Tel: (45) 4525 6392,Fax: (45) 4593 6581,e-mail: alav@fotonik.dtu.dk
}

\begin{abstract}
Metamaterials (MTMs) claim a lot of attention worldwide. Description of the MTMs in terms of effective parameters is a simple and useful tool for characterisation of their electromagnetic properties. So a reliable effective parameters restoration method is on demand. In this paper we report about our activity and advances in the effective properties of metamaterials characterization.

We present here the wave propagation retrieval method in two formulations: for MTMs with linear eigenwaves and for chiral MTMs with circular eigenwaves. The advantages and constraints of the method are noted. The case studies of the negative-index, ultra-high refractive index and chiral MTMs validate the method. Keywords: metamaterial, effective parameters, effective properties, restoration, retrieval, homogenisation
\end{abstract}

\section{INTRODUCTION}

In the recent years, metamaterials (MTMs) attracted a lot of attention worldwide due to their new flashing properties. The range of MTMs applications is very broad, starting from the negative-index superresolution lenses and nanocouplers [1], transformation optics [2] to the MTMs with giant gyrotropic effects, like optical activity [3] and circular dichroism [4]. In the cases when effective parameters (EPs) (refractive index $n$, impedance $Z$, permittivity $\varepsilon$ and permeability $\mu$ ) can be introduced in the correct way [5], a reliable retrieval procedure is on demand. Assigning some certain values to EPs is the simple and useful means, by which the properties of the MTM can be described and the performance of the MTM based devices predicted.

Recently we have proposed a simple retrieval method based on the wave propagation phenomenon [19], which we refer to as the wave propagation retrieval method (WPRM). In this paper we show how WPRM works for negative index and high-index MTMs. We also extend the method to the case of chiral MTMs. The constraints and advantages of WPRM are mentioned as well.

\section{REVIEW OF RESTORATION METHODS}

The existing retrieval methods can be divided into following groups:

1) Reflection/transmission (or S-parameters) based. These methods are based on the reversal of the complex reflection $r$ and transmission $t$ coefficients determined for a slab of compound material. Initially proposed for normal incidence on the isotropic metamaterial [6-7] the procedure was modified for inclined incidence [8], bianisotropic [9-10] and chiral metamaterials [11-13]. The procedure is easy to implement and is widely used, so we refer to it as the standard method (SM).

The SM suffers from the notorious "branch" problem. As we restore refractive index $\operatorname{Re}(n)$ using the inverse cosine function we have an $2 \pi m /(k d)$ ambiguity where $m$ is an integer number, $k$-wavenumber and $d$-slab thickness. The method works well only for thin slabs. However, a thin slab consisting from just a few MTM layers exhibits effective properties, which can be very different from the bulk MTM [14]. Applying the SM to a thick MTM slab is usually problematic. First, different branches of $\operatorname{Re}(n)$ become very close to each other. Second, MTMs are usually absorptive so transmission through the thick slab is very low thus the errors due to numerical noise are large.

2) Field averaging [15-16]. In this case the fields $(\boldsymbol{E}, \boldsymbol{D}, \boldsymbol{H}, \boldsymbol{B})$ in a unit cell are averaged on some surfaces and lines and the effective properties are found according to material relations $\boldsymbol{D}=\varepsilon_{0} \varepsilon \boldsymbol{E}$ and $\boldsymbol{B}=\mu_{0} \mu \boldsymbol{H}$. Determining appropriate surfaces and contours is a non-trivial problem especially when optimizing 3D MTM structures.

3) Quasimode theory [17]. This method is based on the maximization of optical density of states for a metamaterial while changing $\varepsilon$ and $\mu$ of the ambient medium. The method is computationally demanding as it requires 4-parameters optimization for each frequency.

4) Based on wave phenomena. In this case the effective parameters are derived from observation of the wave propagation. For example, Popa and Cummer [18] proposed to match the simulated fields inside and outside of the thin metamaterial slab with the fields theoretically predicted by formulae. Fitting parameters in this case are refractive index and impedance. Due to multiple reflections the standing wave is formed inside the slab so the fitting procedure is complicated.

The WPRM exploits and elaborates ideas proposed in Ref. [18], but it has significant difference. We propose to restore the refractive index $n$ from the wave propagation through a very thick metamaterial slab. In this case we account only one wave propagating in the metamaterial, so the retrieval formulae become easy to use. Such 
retrieval is unambiguous, and the effective parameters derived from the thick layer are bulk automatically. Impedance $Z$ is restored from the input interface reflection that leads to uniqueness of the retrieval and absence of effects caused by finiteness of the structure, like Fabri-Perot resonances.

\section{WAVE PROPAGATION RETRIEVAL METHOD}

The idea of WPRM is to use the spatial dependence of the amplitude and phase of a propagating wave for the EPs restoration. The simulation geometry assumes the MTM slab infinite in $x$ - and $y$-directions and semi-infinite in $z$-direction. The incidence of the wave occurs from vacuum to the MTM. Inside MTM there is only one plane wave (or two waves in the case of chiral MTMs) propagating along the $z$-axis (Fig. 1). The fields have complicated spatial distribution inside the unit cell, so to apply the effective medium description we should abstract out from composite internal structure and regard it as a bulk homogeneous material. Thus we volumeaverage the components of complex field vectors over the unit cell.

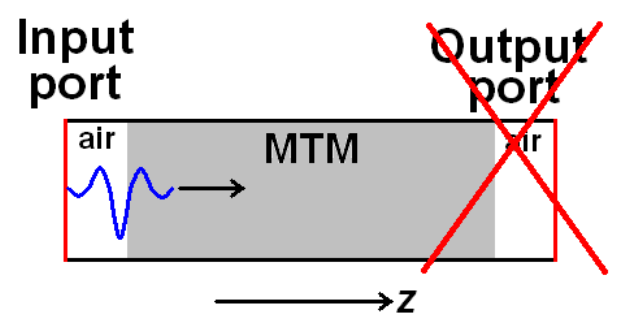

Figure 1. WPRM idea and simulation geometry.

Impedance $Z$ is retrieved unambiguously from the reflection coefficient for the input vacuum-MTM interface:

$$
Z=\frac{1-r}{1+r} \text {. }
$$

The averaged over the $N$-th unit cell electric field inside the thick MTM slab is equal to:

$$
\langle E\rangle_{N}=\frac{\int E_{0} \exp (i k z) d x d y d z}{V} \approx E_{0} \exp \left(i k z_{N}\right),
$$

where $z_{N}=(N-1 / 2) a$ is the coordinate of the $N$-th unit cell centre, $k=k^{\prime}+i k^{\prime \prime}=k_{0}\left(n^{\prime}+i n^{\prime \prime}\right)-$ wavenumber, connected to the refractive index $n=n$ ' $+i n$ ". The refractive index is retrieved according to the following formula

$$
n=-\frac{i}{k_{0}} \frac{\Delta \ln \langle E\rangle}{\Delta z} .
$$

For the case of chiral metamaterials with right and left circular polarised (RCP and LCP, respectively) eigenwaves we should use two components of the electric field $E_{\mathrm{x}}$ and $E_{\mathrm{y}}$ for refractive indices restoration:

$$
\begin{aligned}
& n_{\mathrm{R}}=-\frac{i}{k_{0}} \frac{\Delta \ln \left(E_{\mathrm{x}}-i E_{\mathrm{y}}\right)}{\Delta z}, \\
& n_{\mathrm{L}}=-\frac{i}{k_{0}} \frac{\Delta \ln \left(E_{\mathrm{x}}+i E_{\mathrm{y}}\right)}{\Delta z} .
\end{aligned}
$$

To find out the limitations of WPRM we simulated "the worst case": a homogenous slab with pre-defined refractive index $n$ and impedance $Z=1$ with a perfect mirror (the PEC boundary condition) on the other side of the slab (Fig. 2a). Numerical simulations were performed in the CST Microwave Studio [20] until the total electromagnetic energy stored in the system dropped down to $-80 \mathrm{~dB}$ with respect to the maximum level.

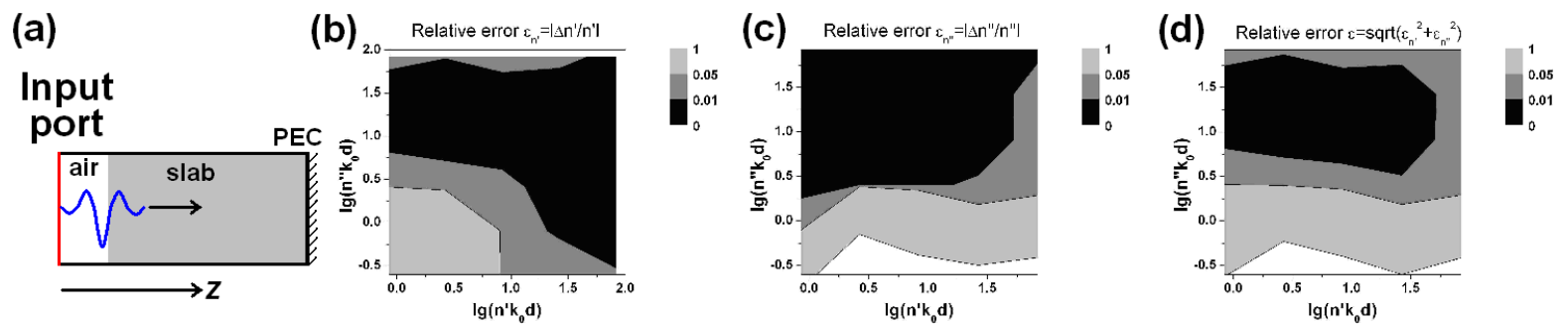

Figure. 2. Configuration of "the worst case” simulation (a). Relative error of (b) $n$ ', (c) $n$ " restoration and (d) total relative error. Black area correspond to the relative error less than $1 \%$. 
It is obvious that not the value of the refractive index itself but the terms in the exponential in (2) play the role in phase and amplitude changes. So we calculated relative error of $n$ restoration for different $n k_{0} d$ and $n$ " $k_{0} d$ (Figs. 2b-2d), where $d$ is the slab thickness. In the case of fast amplitude decay we should consider $d$ not the full thickness of the slab but only until the electric field reaches the noise level., There is an optimal region where the total relative error of both $n^{\prime}$ and $n$ " is less than $1 \%$ (Fig. $2 \mathrm{~d}$ ), which can be approximated with a rectangle. We checked the WPRM for positive index materials but conclusions are valid for negative index materials as well. So we state that WPRM is applicable with a relative error less than $1 \%$ for $-45 \leq n^{\prime} k_{0} d \leq 45$ and $5 \leq n " k_{0} d \leq 56$. In the usual MTM cases these limits are broader as we do not have a perfect reflector at the second interface.

\section{MTM INVESTIGATION WITH WPRM}

To check the validity of the method we studied three cases: negative-index split-cube in cage (SCiC) MTM [21], positive ultra-high refractive index MTM (UHRIMTM) [22] and chiral gammadions MTM (modified version of reported in [11]). The SM was used as a reference. For the case of split-cube in cage and ultrahigh-index MTMs the simulations in CST were performed in the time domain, for the chiral MTM the simulation was done in the frequency domain with periodic boundary conditions.

\subsection{Negative index split-cube in cage MTM}

The remarkable property of the SCiC is its bulkiness: the effective parameters in the negative index region do not depend on the number of layers starting from a single layer [21]. The structure possesses cubic symmetry that insures its polarisation insensitivity. As a drawback, the SCiC has quite low transmittivity and high absorption. Both the WPRM and SM give very similar results (Fig. 3).

(a)

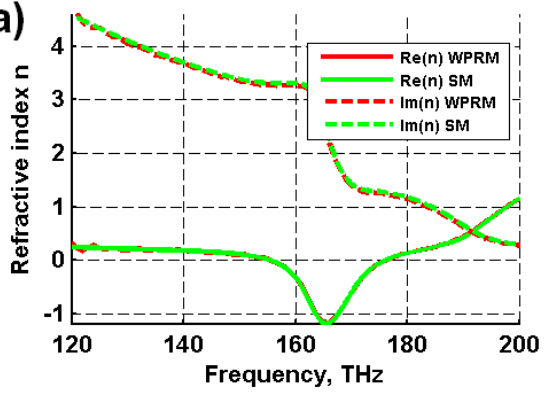

Figure. 3. Split-cube in cage effective parameters:

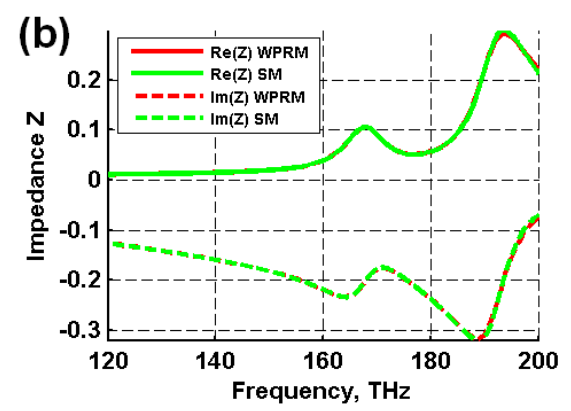

(a) refractive index and (b) impedance.

\subsection{Ultra-high refractive index MTM}

Broadband URIMTM proposed by Shin et al. [22] has low absorption. The idea is to use unit cell consisting of metallic plates connected with wires to obtain the refractive index up to 7. In Ref. [22] there were not specified the exact geometrical parameters of the unit cell so we constructed a similar structure from silver (metal parameters as in Ref. [23]) embedded in silicon $(\varepsilon=12)$.
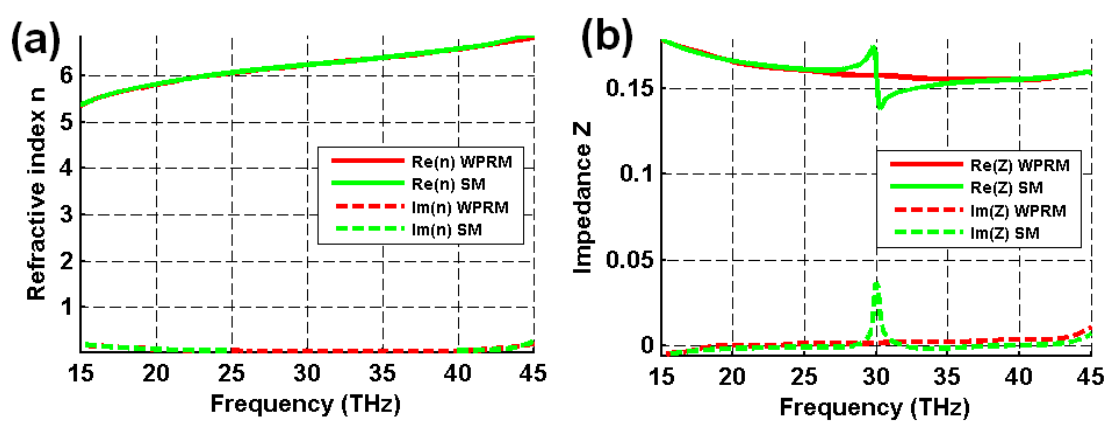

Figure. 4. UHRIMTM effective parameters: (a) refractive index and (b) impedance.

URIMTM shows high refractive index up to 7 in a broad range of frequencies (Fig. 4a). The WPRM and SM results are very close to each other. A kink in the impedance around $30 \mathrm{THz}$ restored by the SM (Fig. 4b) is due to finiteness of the structure. It is worth to mention that $\lg \left(n{ }^{\prime \prime} k_{0} d\right) \approx-0.75$ at frequency $30 \mathrm{THz}$, which is out of the good precision limits defined in Fig. 2 d, but relative difference of WPRM with SM is only $7 \%$. This fact confirms applicability of the WPRM not only to lossy but also to low-loss MTMs. 


\subsection{Chiral gammadions MTM}

The MTM with chiral gammadions is known for providing giant optical activity. For WPRM tests we chose the geometrical parameters of gammadions as in [11] but every second layer twisted by 15 degrees as the initial structure provided very low chirality [11]. The EPs retrieved with the SM and WPRM are shown in Fig. 5. In the low frequency and high frequency region the WPRM results are in a perfect agreement with the SM. In the region $(110 \div 150) \mathrm{THz}$ we observe slow EPs convergence with the number of MTM monolayers and strange values of $n$ from the WPRM. Such EPs behaviour is explained by the strong coupling between the layers. So, such MTM cannot be homogenised here. We can clearly define this region with the WPRM, showing an additional advantage of its usability.
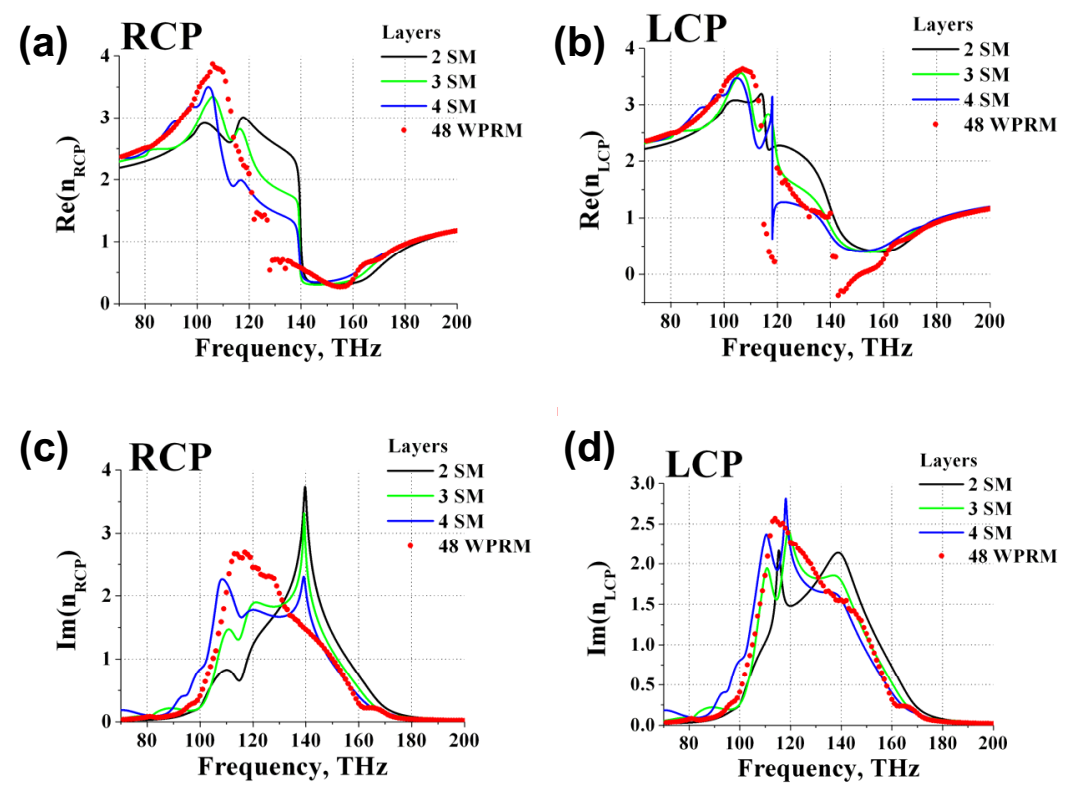

Figure. 5. Effective parameters of the chiral gammadions $M T M: \operatorname{Re}\left(n_{R}\right)(a), \operatorname{Re}\left(n_{L}\right)(b), \operatorname{Im}\left(n_{R}\right)(c), \operatorname{Im}\left(n_{L}\right)(d)$.

\section{CONCLUSIONS}

The WPRM has several clear advantages. First, it does not experience the "branch" problem. Second, WPRM does not suffer from low transmission and numerical noise as it exploits the amplitude and phase distribution inside the structure. The method works well for thick slabs. Third, restoring the EPs with the SM one should always use a broadband excitation source. There is no difference for the WPRM whether to use a broadband or a narrowband source, so one can choose what excitation is more appropriate for a given problem. The WPRM is simple in implementation. Altogether makes the WPRM a useful tool in metamaterials designing.

\section{ACKNOWLEDGEMENTS}

The authors gratefully acknowledge partial support from the Danish Research Council for Technology and Production Sciences via the NIMbus project.

\section{REFERENCES}

[1] J.B. Pendry, Negative refraction makes a perfect lens, Physical Review Letters, vol. 85, p. 3966, Oct. 2000 .

[2] U. Leonhardt, Optical conformal mapping, Science, vol. 312, pp. 1777-1780, Jun. 2006.

[3] M. Decker, M. Ruther, C.E. Kriegler, J. Zhou, C.M. Soukoulis, S. Linden, and M. Wegener, Strong optical activity from twisted-cross photonic metamaterials, Optics Letters, vol. 34, pp. 2501-2503, 2009.

[4] J.K. Gansel, M. Thiel, M.S. Rill, M. Decker, K. Bade, V. Saile, G. von Freymann, S. Linden, and M. Wegener, Gold helix photonic metamaterial as broadband circular polarizer, Science, vol. 325, pp. 15131515, Sep. 2009.

[5] C.R. Simovski, Bloch material parameters of magneto-dielectric metamaterials and the concept of Bloch lattices, Metamaterials, vol. 1, pp. 62-80, Dec. 2007.

[6] D.R. Smith, S. Schultz, P. Markoš, and C.M. Soukoulis, Determination of effective permittivity and permeability of metamaterials from reflection and transmission coefficients, Physical Review B, vol. 65, p. 195104, Apr. 2002.

[7] X. Chen, T.M. Grzegorczyk, B. Wu, J. Pacheco, and J.A. Kong, Robust method to retrieve the constitutive 
effective parameters of metamaterials, Physical Review E, vol. 70, p. 016608, Jul. 2004.

[8] C. Menzel, C. Rockstuhl, T. Paul, F. Lederer, and T. Pertsch, Retrieving effective parameters for metamaterials at oblique incidence, Physical Review B (Condensed Matter and Materials Physics), vol. 77, pp. 195328-8, May. 2008.

[9] X. Chen, B. Wu, J.A. Kong, and T.M. Grzegorczyk, Retrieval of the effective constitutive parameters of bianisotropic metamaterials, Physical Review E, vol. 71, p. 046610, Apr. 2005.

[10] Z. Li, K. Aydin, and E. Ozbay, Determination of the effective constitutive parameters of bianisotropic metamaterials from reflection and transmission coefficients, Physical Review E, vol. 79, p. 026610, Feb. 2009.

[11] C. Menzel, C. Rockstuhl, T. Paul, and F. Lederer, Retrieving effective parameters for quasiplanar chiral metamaterials, Applied Physics Letters, vol. 93, pp. 233106-233106-3, Dec. 2008.

[12] D. Kwon, D.H. Werner, A.V. Kildishev, and V.M. Shalaev, Material parameter retrieval procedure for general bi-isotropic metamaterials and its application to optical chiral negative-index metamaterial design, Optics Express, vol. 16, pp. 11822-11829, 2008.

[13] E. Plum, J. Zhou, J. Dong, V.A. Fedotov, T. Koschny, C.M. Soukoulis, and N.I. Zheludev, Metamaterial with negative index due to chirality, Physical Review B, vol. 79, p. 035407, Jan. 2009.

[14] C. Rockstuhl, T. Paul, F. Lederer, T. Pertsch, T. Zentgraf, T.P. Meyrath, and H. Giessen, Transition from thin-film to bulk properties of metamaterials, Physical Review B, vol. 77, p. 035126, Jan. 2008.

[15] D.R. Smith and J.B. Pendry, Homogenization of metamaterials by field averaging (invited paper), Journal of the Optical Society of America B, vol. 23, pp. 391-403, Mar. 2006.

[16] J. Lerat, N. Malléjac, and O. Acher, Determination of the effective parameters of a metamaterial by field summation method, Journal of Applied Physics, vol. 100, p. 084908, 2006.

[17] S. Sun, S.T. Chui, and L. Zhou, Effective-medium properties of metamaterials: A quasimode theory, Physical Review E, vol. 79, p. 066604, Jun. 2009.

[18] B. Popa and S.A. Cummer, Determining the effective electromagnetic properties of negative-refractiveindex metamaterials from internal fields, Physical Review B, vol. 72, p. 165102, Oct. 2005.

[19] A. Andryieuski, R. Malureanu, and A.V. Lavrinenko, Wave propagation retrieval method for metamaterials: Unambiguous restoration of effective parameters, Physical Review B, vol. 80, p. 193101, Nov. 2009.

[20] "CST Computer Simulation Technology AG."

[21] A. Andryieuski, C. Menzel, C. Rockstuhl, R. Malureanu, and A.V. Lavrinenko, The split cube in a cage: bulk negative-index material for infrared applications, Journal of Optics A: Pure and Applied Optics, vol. 11, p. 114010, 2009.

[22] J. Shin, J. Shen, and S. Fan, Three-dimensional metamaterials with an ultrahigh effective refractive index over a broad bandwidth, Physical Review Letters, vol. 102, p. 093903, Mar. 2009.

[23] G. Dolling, C. Enkrich, M. Wegener, C.M. Soukoulis, and S. Linden, Low-loss negative-index metamaterial at telecommunication wavelengths, Optics Letters, vol. 31, pp. 1800-1802, Jun. 2006. 